\title{
A TAXONOMIC REVISION OF THE ORB WEAVER GENUS ACACESIA (ARANEAE: ARANEIDAE)
}

\author{
By Susan Glueck
}

\begin{abstract}
There are five species of Acacesia which range collectively from southern North America to Argentina. Two are previously known members of the genus, A. cornigera Petrunkevitch and $A$. hamata (Hentz). Three of these are new species: $A$. villalobosi and A. yacuiensis, from southern Brazil, and A. benigna from Bolivia and Peru.
\end{abstract}

\section{INTRODUCTION}

Acacesia is a genus of orb-weaving spiders common and endemic to the Americas, proposed by Simon in 1892. Hentz named the type species Epeira foliata in 1847. The genus contained the single species A. hamata until Petrunkevitch (1925) described A. cornigera. Subsequently published names, illustrations, and descriptions of new species of Acacesia have turned out to be synonyms of the two extant species (Chamberlin and Ivie 1936, Badcock 1932). Levi (1976) redescribed A. hamata in a revision of Nearctic orb weaver genera, mentioning the Neotropical $A$. cornigera in passing. He hypothesized at the time that "there are three or four additional species of Acacesia, all Neotropical and all similar in appearance." The specimens I have examined for the current taxonomic revision corroborate this statement, and I expand the genus to include three new South American species. This study represents an addition to Levi's ongoing project of revising Neotropical orb weavers.

I here report the results of my taxonomic project for which I examined and illustrated over 350 museum specimens. I describe factors I considered in delimiting new species such as their

\footnotetext{
Harvard College, Harvard University, Cambridge, MA 02138

current address: Department of Entomology, Comstock Hall, Cornell University, Ithaca, NY 14853

Manuscript received 7 July 1993.
} 
morphological characters, especially the significant differences in their genitalia, and their geographic distribution.

\section{Materials And Methods}

Most available specimens had been identified only to genus, excepting those members of the two species known when this research commenced in 1989: A. hamata and A. cornigera. The spiders were preserved in $80 \%$ ethanol; some were over fifty years old, so that original color patterns were faded and body parts had darkened with age. They were examined using an American Optical dissecting microscope, on low power $(8.4 \times$ magnification $)$ and medium power ( $48 \times$ magnification). Descriptions and measurements were made following $\mathrm{H}$. W. Levi's suggested guidelines: the color and patterning of the abdomen were noted, along with the shape of the cephalic region, relative distance between eyes, presence of leg macrosetae, etc. The entire thickness of the animal was measured to determine carapace height. When possible the overall length of ten males and ten females of each species was measured, to give some idea of size variation among the species. In addition, in a male and female of each species, the articles of the first leg were measured, and the patella-tibia length of the other legs. Due to the great natural variation within populations, which renders leg length an uninformative character, other specimens were not similarly measured.

Collecting localities were recorded in the computer program Filemaker II, then organized by country, latitude, and longitude. In many cases gazetteers were consulted to provide latitude and longitude when only place names had been indicated. These locations were used to create dot maps on outlines of the North and South American continents to indicate the range of the species.

For each species the genitalia and dorsal pattern were drawn. In males the left palpus was removed and drawn in mesal view, then turned so that the paracymbium could be illustrated. In females the epigynum was drawn in ventral and posterior (up-turned) view. In some cases drawings of the dorsal pattern were composites of several specimens to take individual variations into account.

New species were identified on the basis of genitalic and dorsalpattern differences. Further specimens were compared with draw- 
ings of the holotypes, and double-checked by H. W. Levi before locality information was recorded.

\section{RESULTS}

I added three new species, Acacesia villalobosi, A. yacuiensis, and $A$. benigna, to the two previously known members of the genus, A. cornigera Petrunkevitch and A. hamata (Hentz). The new species were collected solely on the South American continent, whereas A. hamata and $A$. cornigera range further north along the isthmus of Panama and into North America.

\section{DISCUSSION}

In 1892 Simon split up the huge genus Epeira (the name previously used by Hentz) into a number of genera including Acacesia. To single out individual species I relied upon differences in genitalia. Levi (1976) had noted in his diagnosis "In Acacesia alone among related genera the paracymbium is modified, and not simply hook-shaped". The paracymbium ornaments the posterior edge of the base of the cymbium which surrounds the palpus ( $\mathrm{P}$ in Figs. 8, 16). I used the evidence of variations of the paracymbium to separate males of three new species: A. yacuiensis, A. villalobosi, and A. benigna. Otherwise, the males of the genus showed marked uniformity in the design of the palpus itself. The main obvious differences were in the shape and proportions of the median apophysis, the large structure (two-pronged in this genus; $M$ in Fig. 1) that may grasp the scape of the epigynum in maneuvering the male's sperm-transferring embolus into contact with the females' sperm duct (Foelix 1982).

An examination of the mapped location of specimens demonstrates the overlap of the various species of the genus, for the most part sympatric. It is possible that strong genitalic (and likely unknown behavioral) differences have maintained reproductive isolation allowing for sympatric speciation, despite the notable homogeneity of dorsal pattern and body form. None of the species were abundant within the lower Amazon basin area; topographic maps indicate a bare region in the watershed. It is possible either that the habitat is different from that usually frequented by the genus, perhaps due to its lower elevation or higher annual rainfall, 
or that the topography and vegetation of the region effectively bars collectors from entering. More information about the ecology of collection sites would help to clarify this situation. Preference for certain collection sites certainly has affected the geographic data for other members of the genus. A. villalobosi is a case in point: most of the specimens were collected within a short distance of two major Brazilian cities, São Paulo and Porto Alegre. This suggests that collectors restricted their efforts to the most accessible regions. The relative paucity of specimens also makes it difficult to make any generalizations about the range of this species. The situation is similar for A. yacuiensis and A. benigna. A more complete biogeographic picture of Acacesia awaits further fieldwork, especially in those regions which appear devoid of specimens.

\section{Acacesia Simon}

Acacesia Simon, 1892: 795. Type species by original designation and monotypy Epeira foliata Hentz (= E. hamata Hentz). The name is feminine (Levi 1976: 374).

Description (see also Levi, 1976). Dorsum marked with a dagger shape, which is outlined in black and surrounded by a triangular folium. Parallel rows of orange-brown dots extend posteriorly on either side of the dagger (Fig. 6). Depending on state of preservation and regional or intraspecific variation, branching pattern may appear extending from the tip of the dagger, often terminating in a straight line. Other variations: presence of dark gray shoulder patches and coloration within and outside folium. These variations do not appear marked enough to allow for distinguishing species, although familiarity with specimens may reveal a gestalt that is clear at least to the researcher.

Width of cephalothorax directly behind the posterior lateral eyes less than or equal to one-half the width of the carapace at its widest part (Figs. 6, 11, 14, 19, 22, 25, 30). Males with macroseta on the fourth coxa and trochanter, and one prominent spur, surrounded by shorter and thinner macrosetae, on the swollen second tibia. One macroseta on the palpal patella. First coxa laterally elongated forming a hook on the outside corner. Male palpus with bifurcate median apophysis ( $\mathrm{M}$ in Fig. 1); length of the tines of this apophysis vary according to species, as does the shape of the hinge where the apophysis connects to the palpus. Embolus resem- 
bling a bird's head with a curved and pointed beak, covered by a transparent lamella (E in Fig. 1). Paracymbium varies interspecifically in size and shape (Figs. 8, 16, 21, 27,33). As with the paracymbia, the sclerotized edges of the lateral plates of the underside of the female epigynum show great interspecific variation and range of complexity. Scape of the epigynum always extends posteriorly from the base (Fig. 4).

Only the natural history of A. hamata is known in any detail, as the other species were identified solely from preserved museum specimens. Thus natural history is unavailable for the individual species diagnoses that follow. Due to the overall similarity of dorsal pattern within the genus, only striking interspecific variation is noted in the species diagnoses.

Diagnosis. Hentz's description of Epeira foliata (1847: 475) serves to diagnose the genus: "abdomen ovate terminating in a joint, with waved black lines, two external almost meeting at the apex, two internal meeting before or near the middle." Much later Kaston (1978: 144) also noted hamata's triangular folium with "undulating margins." The genus is distinguished from its close relatives Ocrepeira and Cyclosa by the foliate dorsal pattern (Fig. 6) which is largely invariant from species to species (Petrunkevitch, 1925, noted its "generic value"), as well as by the oval abdomen, which forms a shield shape in Ocrepeira and is often marked by tubercles in Cyclosa. In Acacesia the abdomen is an ovoid, rounded-off diamond shape often with "shoulder" humps and a small hump at the extreme anterior end (Figs. 6, 11, 14, 19, $25,30)$. The spinnerets are at the posterior end of the abdomen (Fig. 31), as in Ocrepeira, but differing from Cyclosa where they lie in the center, beyond which extends a "tail."

Males of related genera have rows of macrosetae on the second tibia, as opposed to Acacesia's main enlarged spur (Fig. 3). The embolus varies little within the genus, contrasted, for example, with Cyclosa, some species of which have extremely long emboli curving around the palpus. The paracymbium has evolved different ornamentation (P in Fig. 2; Figs. 8, 16, 21, 27, 33), whereas in presumed sister genera it appears constant as a simple club-shaped attachment. (Within Acacesia at least, it proves useful as a determinant of species). The extended epigynal scape occurs also in Ocrepeira, but overlaps the base in Cyclosa. Whereas in Ocrepeira 
the scape may be twice the length of the base, reaching almost to the spinnerets, in Acacesia the scape is rarely much longer than the base (Figs. 4, 9, 12, 17, 23, 28).

White spots on the venter appear constant only in A. villalobosi (Fig. 31), where they are large enough to be visible without the aid of a microscope, and in A. yacuiensis. The other Acacesia species appear to have white stripes extending the length of the venter and surrounding a gray central patch.

\section{Misplaced Acacesia.}

Two single specimens do not fit into any of the species: one from La Paz, Bolivia (AMNH) may be a penultimate ready to molt, and one female from Alto Salimões (MCN 8821) which remains puzzling. It may be a cornigera.

Epeira vegeta Keyserling, 1865: 819, pl. 19, figs. 31-34, ơ, ᄋ, was placed in Acacesia by Simon, 1895: 789, figs. 858-859, ơ, but was placed by F. P.-Cambridge in Eustala, 1904: 509, pl. 48, figs. 16,17, \&, ơ. Chickering (1955: 511) redescribed and illustrated the Central American Eustala species.

\section{Key to Female ACaCEsia Species}

1. Base of epigynum a trapezoid with posterior edge wider than anterior and wide spatulate scape (Fig. 28); sclerites in posterior view of epigynum like reversed quotation marks (Fig. 29); southeastern Brazil (Map 1)...................................villalobosi

-. Base of epigynum and scape otherwise (Figs. 4, 9, 17, 23); posterior sclerites otherwise (Figs. 5, 10, 18, 24).

2. (1) Scape with lip on posterior end (Fig. 9) and anteriorly sclerotized median plate in posterior view (Figs. 10, 13); central Mexico to southern Brazil (Map 1) cornigera

-. Scape without lip (Figs. 4, 17, 23); median plate in posterior view otherwise (Figs. 5, 18, 24)

3. (2) Scape shorter than base (Fig. 17); in posterior view sclerotized median edges of lateral plates kidney-shaped with ridge or wrinkle at anterior end (Fig. 18); southeastern Brazil, northeastern Argentina (Map 1) yacuiensis

-. Scape approximately as long as base; base with two visible dark spots (Figs. 4, 23); lateral plates otherwise (Figs. 5, 24) .4 


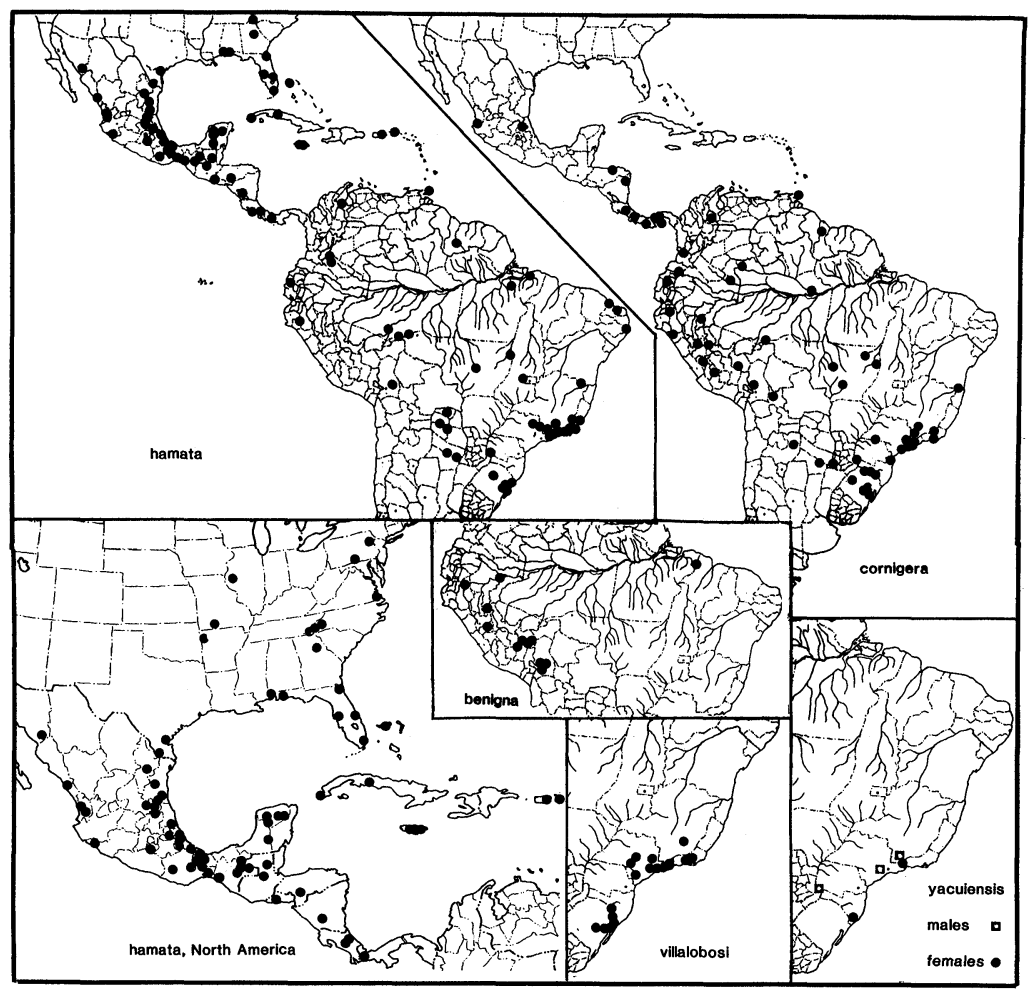

Map 1. Distribution of Acacesia species.

4. (3) Visible part of median plate hourglass-shaped (Fig. 5); North and South America (Map 1) hamata

-. Median plate otherwise; sclerotized lateral plates resemble Mickey Mouse hat with big ears (Fig. 24); Peru, Brazil, Bolivia (Map 1) benigna

\section{Key to Male Acacesia Species}

1. Paracymbium wider than high (Figs. 8, 21, 33) .....................2

-. Paracymbium higher than wide (Figs. 16, 27) .......................4

2. (1) Paracymbium with distal lobe, otherwise unadorned; margin of proximal lobe convex (Fig. 8); North and South America (Map 1) hamata 
- Paracymbium with additional appendage or ornamentation, like thorn or lip (Figs. 21, 33) .............................................

3. (2) Thorn-shaped projection of paracymbium hanging over distal lobe. Margin of proximal lobe concave (Fig. 21); southeastern Brazil (Map 1) yacuiensis

-. No such projection; instead, distal lobe of paracymbium with folded lip at edge, overlapped by partly transparent proximal lobe. Transparent margin of proximal lobe straight, opaque margin concave (Fig. 33); southeastern Brazil (Map 1) ...villalobosi

4. (1) Distal lobe of paracymbium longer than proximal lobe, both anteriorly directed (Fig. 16); central Mexico to southern Brazil (Map 1) cornigera

-. Distal lobe of paracymbium and proximal lobe approximately the same length, both anteriorly directed (Fig. 27); Peru, Brazil, Bolivia (Map 1) benigna

\section{SPECIES DESCRIPTIONS}

Abbreviations: AME, anterior median eyes; PME, posterior median eyes; LE, lateral eyes. For museum collection abbreviations refer to the Acknowledgements section.

Figure 1. Expanded left palpus of male A. hamata. Cali, Colombia (MCZ). M, median apophysis; $\mathrm{C}$, conductor; E, embolus; R, radix; P, paracymbium; CY, cymbium; $\mathrm{L}$, terminal apophysis or embolus lamella.

Figure 2. Palpus of male A. cornigera. $\mathrm{P}=$ paracymbium. Panama (PMY). Figure 3. Left second tibia of male A. cornigera, posterior. Panama (PMY). Figs. 1-3 by Levi.

Figures 4-8. Acacesia hamata (Hentz). 4-6, female. 4. Epigynum, ventral. Chiapas, Mexico (MCZ). 5. Epigynum, posterior. Same. B, base of epigynum; S, scape of epigynum; MP, median plate; LP, lateral plate. 6. Dorsal. Quezaltepeque, El Salvador (CAS). 7, 8, male, left palpus. 7. Mesal. Cali, Colombia (MCZ). 8. Paracymbium. Naciemente del Río Frio, Tamaulipas, Mexico (MCZ).

Figures 9-16. A. cornigera Petrunkevitch. 9-11, female. 9. Epigynum, ventral. Barro Colorado Island, Canal Zone (MCZ). 10. Epigynum, posterior. Near Gamboa, Panama (MCZ). 11. Dorsal. Sirena, Osa Peninsula, Costa Rica (MCZ). 12. Epigynum, ventral. Aparo, São Paulo, Brazil (MZSP). 13, Epigynum, posterior. Aparo, São Paulo, Brazil (MZSP). 14. Dorsal. Aparo, São Paulo, Brazil (MZSP). 15, 16, male, left palpus. 15, Mesal. Barro Colorado Island, Canal Zone (MCZ). 16. Paracymbium. Same.

Scale lines. $1.0 \mathrm{~mm}$, genitalia $0.1 \mathrm{~mm}$. 

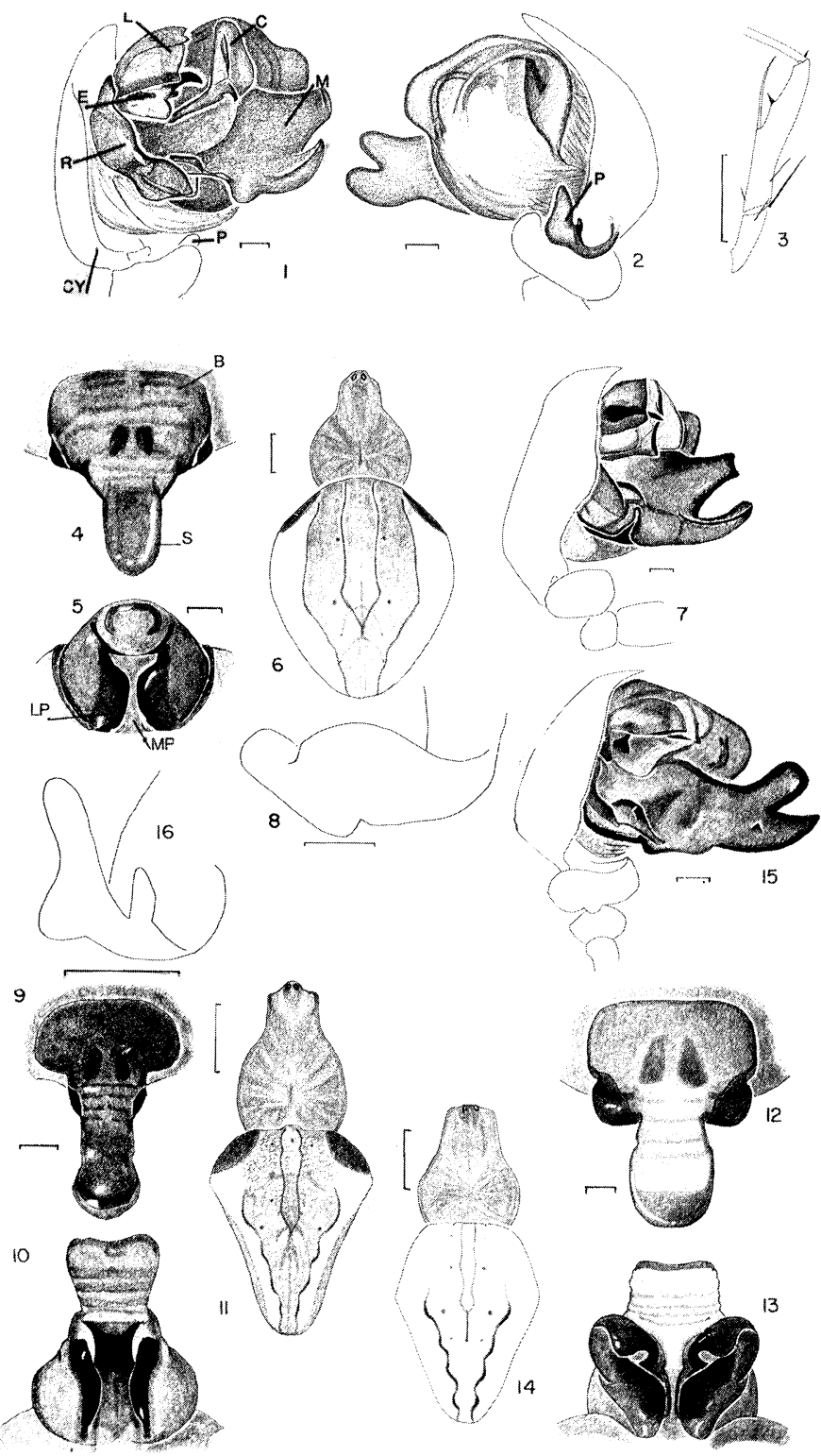


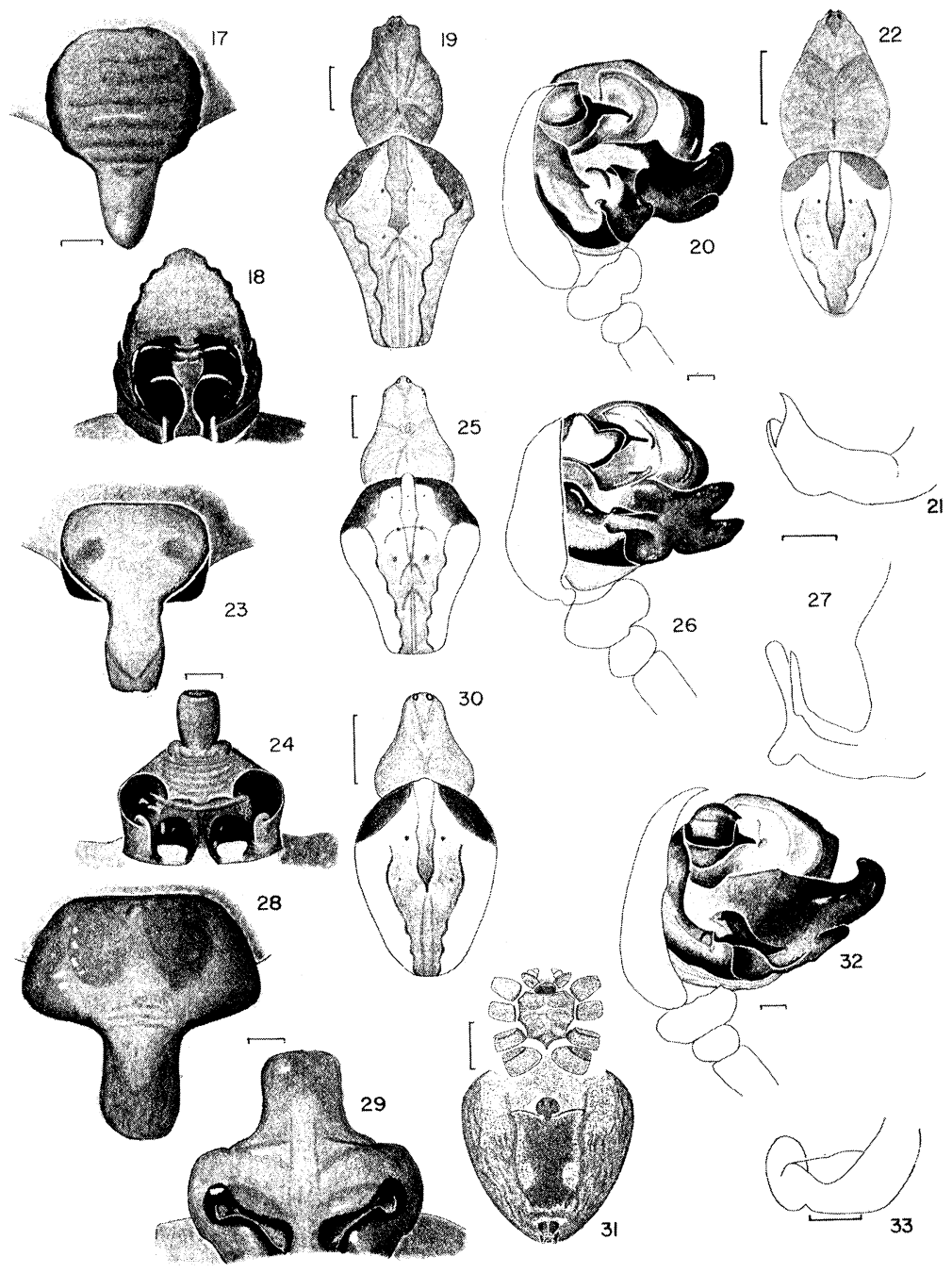




\section{Acacesia hamata (Hentz)}

Figures 1, 4-8

Epeira hamata Hentz, 1847: 474, pl. 31, fig. 10, ơ. Specimen from Alabama in Bos. Soc. Natur. Hist., destroyed (Hentz 1875 ed. Burgess, vii).

Epeira foliata Hentz. 1847: 475, pl. 31, fig. 14, o. Female specimen from Alabama in Bos. Soc. Nat. Hist., destroyed (Hentz 1875 ed. Burgess, vii). (Not E. foliata C. L. Koch, 1845). Emerton, 1884: 318, pl. 34, fig. 10, o, pl. 37, figs. 6-10, ơ. McCook, 1894: 154, pl. 4, figs. 7, 8, ᄋ, ơ. F. P.-Cambridge, 1904: 502, pl. 48, figs. 1, O', and 2, o.

Epeira folifera Marx, 1890: 545, 593. New name for foliata Hentz, preoccupied.

Araneus hallucinor Petrunkevich, 1911: 296. New name for Epeira hamata Hentz, thought preoccupied by Araneus hamatus Clerck, (= Singa hamata).

Acacesia foliata: - Comstock, 1912: 509, figs. 546, 548, o, 547, ơ. Comstock, 1940: 522-3, figs. 546, 548, ㅇ, 547, ơ. Bonnet, 1955: 122.

Acacesia lanceolata Badcock, 1932: 20, fig. 14, ᄋ. Type specimen from Nanahua, Paraguay; in BMNH, examined. (Synonymized in Roewer, 1942, Bonnet, 1955).

Figures 17-22. A. yacuiensis. new species. 17-19, female. 17. Epigynum, ventral. Municipio de Itu, São Paulo, Brazil (AMNH). 18. Epigynum, posterior. Same. 19. Dorsal. Composite drawing of specimens from Arroyo Yacui, Parque Nacional Iguazu, Misiones Province, Argentina (M.E. Galiano). 20-22, male. 20. Left palpus, mesal. Baixo Jordão, Siderópolis, Santa Catarina, Brazil (MCN). 21. Left palpus, paracymbium. Same. 22. Dorsal. Same.

Figures 23-27. A. benigna. new species. 23-25, female. 23. Epigynum, ventral. Zona Reservada de Manu, Puesto Vigil, Pakitza, Madre de Dios, Peru (USNM).

24. Epigynum, posterior. Same. 25. Dorsal. Puesto de Vigilancia 22 "Falso Paquisha" (sic), Alto Río Comaina, Amazonas, Peru (MHNSM). 26, 27, male, left palpus. 26. Mesal. Est. Biol. Beni, Beni, Bolivia (USNM). 27. Paracymbium. Same.

Figures 28-33. A. villalobosi. new species. 28-31, female. 28. Epigynum, ventral. Serra dos Orgãos, Rio de Janeiro, Brazil (MCZ). 29. Epigynum, posterior. Same. 30. Dorsal. Fazenda Sta. Maria Amparo, São Paulo, Brazil (MZSP). 31. Abdomen, ventral. Canela, Rio Grande do Sul, Brazil (MCN).

32, 33, male, left palpus. 32. Mesal. Campo do Jordão, São Paulo, Brazil (MZSP).

33. Paracymbium. Same.

Scale lines. $1.0 \mathrm{~mm}$, genitalia $0.1 \mathrm{~mm}$. 
Acacesia folifera: -Roewer, 1942: 763.

Acacesia hamata: - Bryant, 1945: 364. Kaston, 1948: 235, fig. 705, 726, ơ, 725, ९. Levi, 1976: 376, figs. 74-87, ᄋ, ơ.

Araneus nigrolineatus di Caporiacco, 1955. Female holotype from

Rancho Grande, Aragua, Venezuela in UCV. NEW SYNONYMY.

Description. Female, from Nantahala Gorge, North Carolina, MCZ. Carapace yellow-brown. Clypeus white. Chelicerae light brownish yellow. Labium light yellow-white, endites light brownish yellow. Sternum light yellow-white. Coxae light yellow-brown; legs yellow-brown. Dorsum of abdomen light gray-brown, sides off-white, venter tan. PME 1.1 diameters of AME, LE 0.5 diameters. AME 2.2 diameters apart, 2.5 from LE. PME 1.0 diameters apart, 3.5 from LE. LE less than 1 diameter apart. Total length 5.4 $\mathrm{mm}$. Carapace length $2.2 \mathrm{~mm}$, width 1.9 , height 1.4 . First femur $3.9 \mathrm{~mm}$, patella-tibia 4.4 , metatarsus 3.2 , tarsus 1.0. Length of first leg 12.5. Second patella-tibia $4.3 \mathrm{~mm}$, third 1.8, fourth 3.0.

Male from Lago Calima, Depto. Valle, Colombia, 1,400 m elev., MCZ. Color as in female. PME 0.6 diameters of AME, LE 0.5 diameters. AME 2.0 diameters apart, 1.0 from LE. PME 2.0 diameters apart, 3.0 from LE. LE less than 1 diameter apart. Total length $4.3 \mathrm{~mm}$. Carapace length $1.9 \mathrm{~mm}$, width 1.8 , height 1.1 . First femur $2.3 \mathrm{~mm}$, patella-tibia 3.5 , metatarsus 2.3 , tarsus 0.5 . Length of first leg 8.6. Second patella-tibia $2.2 \mathrm{~mm}$, third 1.2, fourth 1.8 .

Variation. Total length of ten females 5.3 to $8.6 \mathrm{~mm}$, of ten males, 3.6 to 4.4 .

Diagnosis. Specimens of A. hamata lack distinct white spots on the venter of the abdomen. The straight-line folium pattern (Fig. 6) is characteristic and differs from the more sinuous forms on the backs of A. cornigera, A. yacuiensis, and A. villalobosi. A. hamata's abdomen is smoothly oval, not with protruding "shoulders" above a constriction, as is common in A. cornigera and $A$. yacuiensis. Almost total absence of intraspecific variation in the paracymbium (Fig. 8) makes it a good character by which to identify males. It is the simplest form in the genus, with proximal and distal lobe of equal height, and with the proximal lobe forming a convex curve leading into the distal lobe. In the females the edges of the lateral plate of the epigynum overlap the median plate to form an hourglass shape (Fig. 5). 
Natural History. See Levi 1976.

Distribution. The species ranges from eastern Pennsylvania to western Arkansas, south into Florida and the Bahamas, through Mexico into northern Argentina.

Specimens examined. ARGENTINA Chaco: Presidencia Roque Saenz Peña (MACN); Selvas del Río de Oro (Galiano). Misiones: Eldorado (AMNH). BAHAMAS South Bimini (AMNH); Freeport (AMNH). BOLIVIA La Paz: Yungas, Puente Villa (IRSNB). BRAZIL Amapá: Oiapoque (AMNH). Espírito Santo: Guarapari (MZSP). Goiás: São Francisco, Jaraguá (MZSP); Fazenda Monjolinho, Corumbá (MZSP); Jataí (AMNH). Mato Grosso: Rosário Oeste (AMNH); Fazenda Beija-Flor (MZSP). Minas Gerais: Barbacena (AMNH); Pedra Azul (AMNH); Viçosa (AMNH); Lavras (MCZ); Varginha (AMNH). Pará: Belém, Val-de-Cães (AMNH). Paraíba: Indepência (MCZ). Paraná: Rolândia (AMNH). Pernambuco: Recife (MNRJ). Rio de Janeiro: Silvestre (AMNH); Floresta dos Macacos (AMNH); Santa Maria Madalena (AMNH); Santo Antônio do Imbé (AMNH); Silva Jardim (AMNH); Nova Iguaçu, Miguel Couto (AMNH); Reprêsa Rio Grande (AMNH); Mangaratiba (AMNH); Angra dos Reis (MZSP); São Conrado (MCZ); Niterói (MNRJ). Rio Grande do Norte: Fazenda Canaan (MZSP); Fazenda Camão, Macau (MZSP). Rio Grande do Sul: S. Heupolblo (MZSP); Morro São Pedro (MCN); Tapera (MNRJ); Muçum (MCN); Encantado (MCN); Roca Sales (MCN); Montenegro (MCN); General Câmara (MCN); Triunfo (MCN); Mono Alto, Gravataí (MCN); Porto Alegre (MCN); Viamão (MCN); Santa Maria (MCN); Taquara (MCN); Butiá (MCN). São Paulo: Fazenda João, Inda Verde (MZSP); Itaguerê, Nova Europa (MZSP); Estaç, Exper., Pirassununga (MZSP); Ilha dos Buzios (MZSP); Barueri (MZSP); Ubatuba (MZSP); Municipio de Itu, Fazenda Pau d'Alho (AMNH); São Roque (AMNH). BRITISH VIRGIN ISLANDS Great Thatch Island: Tortola (AMNH). COLOMBIA Antioquia: La Estrella (MCZ). Cundinamarca: Monte Redondo (MCZ). Magdalena: Pueblo Bello, Sierra Nevada de Santa Marta (AMNH). Valle: Lago Calima (MCZ); Río Jamundí (MCZ); Cali (MCZ). COSTA RICA Cartago: Turrialba (AMNH). San José: San José (AMNH); Carillo (MCZ). Guanacaste: Cañas: La Pacífica (MCZ). CUBA Pinar del Río: Peninsula de Guanahacabibes (AMNH). Cienfuegos: Soledad (AMNH). ECUADOR Guayas: $8 \mathrm{mi}$. S 
Manglaralto (CAS). GUATEMALA Nebaj (AMNH); Tikal (MCZ). Petén: Santa Elena (AMNH). EL SALVADOR Quezaltepeque, $\$$ (CAS). GUYANA Yupukari, Rupununi River (AMNH). HONDURAS Zamorano (AMNH). JAMAICA $14 \mathrm{mi}$. E Kingston (AMNH); Mandeville (AMNH); Sandy Ground (AMNH); Grove Place (AMNH); 3 mi. N Spanish Town (AMNH). MEXICO Campeche: Campeche (MCZ). Chiapas: $15 \mathrm{mi}$ NW Arriaga (AMNH); Palenque ruins (MCZ). Colima: Armería (AMNH). Morelos: Cuernavaca (AMNH). Nayarit: Jalisco (AMNH); $26 \mathrm{mi}$. N Rosamorada (AMNH); $20.5 \mathrm{mi}$. NW Tepic, ९ (CAS). Nueva León: Montemorelos (AMNH). Oaxaca: $2 \mathrm{mi}$. SE Niltepec (AMNH); Palomares (MCZ). Puebla: Villa Juarez (AMNH). San Luis Potosí: Picolo (AMNH); Tamazunchale (AMNH); Huichihuayán (AMNH); El Pujal (AMNH); Covadonga, S Valles (AMNH); $28 \mathrm{mi}$. E Ciudad del Maíz (AMNH); $14 \mathrm{mi}$. W of Xilitla (CNC). Sinaloa: Villa Unión (AMNH). Sonora: Minas Nuevas (AMNH). Tamaulipas: $22 \mathrm{mi}$ SSW Mante (AMNH); Hidalgo (AMNH); 6 mi. n C. Victoria (CAS). Veracruz: Paso Real, Río Tonto (AMNH); $22.5 \mathrm{mi}$ NW Ciudad Alemán (AMNH); Lago Catemaco (AMNH); Orizaba (MCZ); Tierra Colorada (AMNH); Conejos (AMNH); 4 mi. SE Jalapa (AMNH); Papantla de Olarte (AMNH); La Buena Ventura (AMNH). Yucatan: Grutas de Loltun (MCZ); Uxmal (AMNH); Valladolid (AMNH); Pisté (AMNH); Yaxcopoil (MCZ); Mérida (AMNH); Colonia Yucatan (AMNH); Yaxché (AMNH). PANAMA Chiriquí: Volcán, Bambito (AMNH). NICARAGUA: Leon, Los Lechecuagos (J. M. MAES). PARAGUAY Alto Paraná: Taquarazapa (AMNH). Chaco: Mission Nuevo Tribu (IRSNB). Nueva Asunción: Estancia "La Madlon" km 470 (IRSNB). Boquerón: Transchaco (IRSNB); (?)Teniente Espinola (IRSNB). PERU Lamboyeque: Olmos (AMNH). PUERTO RICO Río Piedras (AMNH). TRINIDAD St. Augustine (AMNH). UNITED STATES Alabama: Chickasaw (MCZ). Arkansas: Washington Co., Cove Cr. Vall., S. Prairie Grove (MCZ). Florida: Miami (MCZ); Sebastian (MCZ); Dunedin (MCZ); Pensacola (MCZ); Marietta (MCZ). Georgia: Clarke Co., Athens (MCZ). Illinois: La Prairie, Adams Co. (MCZ). Missouri: Galena (MCZ). North Carolina: Canton (MCZ); Nantahala Gorge (MCZ); Balsam Gap (MCZ). Pennsylvania: Gettysburg (MCZ); Newfoundland Gap (MCZ). Texas: Hidalgo Co. (MCZ); Sinton, 
San Patricio Co. (MCZ). Virginia: Portsmouth (MCZ). VENEZUELA Aragua: Rancho Grande (AMNH). Caracas: Los Tejes (ZMK), El Valle nr. Caracas (MNRJ).

\section{Acacesia cornigera Petrunkevitch}

Figures 2-3, 9-16; Map 1

Acacesia peruviana Chamberlin, 1916: 244, pl. 18, fig. 6, o. Damaged immature holotype from San Miguel, Peru in MCZ. DOUBTFUL NEW SYNONYMY.

Acacesia cornigera Petrunkevitch, 1925: 117, figs. 32, 34, 40, 41, o, 33, 35, 36-39, ơ. Female, male and juvenile syntypes from San Lorenzo, Panama in PMY. Examined by H. W. Levi, October 1973. Roewer, 1942: 763. Bonnet, 1955: 122. Levi, 1976: 376.

Acacesia alticeps Chamberlin and Ivie, 1936: 54, fig. 140, ․ Female holotype from Barro Colorado Island, Panama in AMNH. Roewer, 1942: 763. Bonnet, 1955: 122. NEW SYNONYMY.

Acacesia spinulosa Mello-Leitão, 1948: 158, fig. 6, @. Female holotype from Moraballi River, Guyana, in BMNH. Examined by Levi. Brignoli, 1983: 254. NEW SYNONYMY.

Acacesia pentagona di Caporiacco, 1954: 86, fig. 15, ○. Female holotype from Charvein, French Guiana in MNHN. Examined by Levi. NEW SYNONYMY.

Description. Female, from Sirena, Osa Peninsula, Puntarenas Prov., Costa Rica. MCZ. Carapace light yellow-brown. Clypeus light yellow. Chelicerae light yellow. Labium light yellow, endites very light yellow. Sternum very light yellow. Coxae light yellow; legs yellow-tan. Dorsum of abdomen off-white with gray shoulder patches, sides dark gray-brown, venter dark gray-brown. PME 1.0 diameters of AME, posterior LE 0.5 diameters, anterior LE 0.6 diameters. AME 1.6 diameters apart, 3.4 from LE. PME 1.6 diameters apart, 4.1 from LE. LE less than 1 diameter apart. Total length $5.2 \mathrm{~mm}$. Carapace length $2.2 \mathrm{~mm}$, width 1.7, height 1.2. First femur $3.5 \mathrm{~mm}$, patella-tibia 4.7 , metatarsus 2.8, tarsus 0.94 . First leg length overall 11.94. Second patella-tibia $4.0 \mathrm{~mm}$, third 1.9 , fourth 2.6 . 
Male, from Summit, Panama, MCZ. Color as in female. PME 0.9 diameters of AME, LE 0.5 diameters. AME 1.8 diameters apart, 1.4 from LE. PME 1.1 diameters apart, 2.5 from LE. LE less than 1 diameter apart. Total length $3.8 \mathrm{~mm}$. Carapace length 1.8 $\mathrm{mm}$, width 1.5 , height 0.79 . First femur $3.3 \mathrm{~mm}$, patella-tibia 3.6 , metatarsus 2.2, tarsus 0.66. First leg length overall 9.76. Second patella-tibia $2.5 \mathrm{~mm}$, third 1.2 , fourth 2.1 .

Variation. Total length of ten females 5.4 to $9.0 \mathrm{~mm}$, of ten males, 3.3 to 4.4. Variation of epigyna illustrated by Figs. 9, 10, 12,13 . There is variation in length of the scape and how much of the underside of the median plates is visible in ventral view. Most Brazilian specimens are closer to Figures 12, 13 (from São Paulo State, Brazil) and more northern specimens as in Figures 9, 10 (made from a female from Panama). Figures 15, 16 were made from a male from Panama.

Diagnosis. A. cornigera vies with A. hamata as the most commonly collected species. A. hamata's folium possesses almost straight lines, compared to A. cornigera where they are markedly more sinuous (Fig. 11). The broad distal lobe of the paracymbium is always longer than the proximal lobe, which varies regionally in length from nearly invisible to half the height of the distal lobe (Figs. 2, 16). A. cornigera's scape is much longer relative to $A$. hamata's and has a lip on its posterior edge (Fig. 9). The length of the scape also varies throughout the distribution of the species, ranging from one to one and one-half times the length of the base. The tines of the median apophysis (Fig. 15) are equal in length.

Distribution. A. cornigera ranges from central Mexico to southern Brazil, and is found on both sides of the Andes.

Specimens examined. ARGENTINA Chaco: Presidencia Roque Saenz Peña (MACN); Selvas del Río de Oro (MEG). Salta: Tabacal (MACN). Misiones: Eldorado (AMNH); Dept. San Antonio, Arroyo El Central (MEG). BAHAMAS Grand Bahama Island: Freeport (AMNH). BOLIVIA La Paz: Yungas, Chulumani (IRSNB); Serrania Bellavista. $47 \mathrm{~km} \mathrm{~N}$. Caranavi. 1,350 m, nr. campsite, small clearing in virgin upper tropical zone forest, $\odot$ (AMNH). BRAZIL Amazonas: Manaus (INPA). Bahia: Camacã (MCN). Rio de Janeiro: Nova Iguassu, Miguel Couto (AMNH); Santa Maria Madelena (AMNH); Morro de São João, Copacabana (AMNH). Rio Grande do Sul: Montenegro (MCN); Novo Hamburgo 
(MCN); São Borja, Garruchos (MCN); Aguas Belas, Viamão (MCN); Triunfo (MCN); Parque Estad. de Nonoai (MCN); Tenente Portela (MCN). São Paulo: Guarulhos (MCZ); Fazenda Poço Grande Juquiá (MZSP); Fazenda Nossa Senhora da Encarnação (MZSP); Serra Gusperurrí, Mongaguá (MZSP); Castilho (MZSP); Engenheiro Marsilac (MZSP); Municipio da Itu, Fazenda Pau d'Alho (AMNH). Minas Gerais: Lavras (MCZ). Mato Grosso: Parque Nac. Xingu, Jacaré (AMNH); $260 \mathrm{~km} \mathrm{~N}$ Xavantina (MCZ); Barra dos Bugres (MNRJ); Rio Culuene, (MNRJ); Utiariti (MZSP). Paraná: Rolândia (AMNH); Tijucas do Sul (MCN). Santa Catarina: Pinhal da Serra (AMNH). BRITISH GUIANA Upper Essequibo River (AMNH). COLOMBIA Amazonas: Río Pira, Apaporis, $0^{\circ} 25^{\prime} \mathrm{S}, 70^{\circ} 15^{\prime} \mathrm{W}$ (CAS). Valle: Cali (MCZ). Nariño: Barbacoas (MCZ). Vaupés: Mitú (MCZ). César: $8 \mathrm{~km} \mathrm{SE}$ Socorpa Mission, Sierra de Perijá (AMNH). COSTA RICA San José: San José (MCZ, AMNH); San Isidro del General (MCZ). Heredia: La Selva, nr. Puerto Viejo (MCZ). Puntarenas: Osa Peninsula, Sirena (MCZ). ECUADOR Pichincha: $35 \mathrm{~km} \mathrm{NW}$ Santo Domingo de Los Colorados (AMNH). Zamora-Chinchipe: Jumboé River (MCZ); Zamora (MCZ). HONDURAS Pine Ridge Peak (MCZ). MEXICO Nayarit: $5 \mathrm{mi}$. NE San Blas (AMNH). San Luis Potosí: Tamazunchale (AMNH). Chiapas: Ocosingo Valley, Finca El Real (AMNH). NICARAGUA Masawas: Waspuc River (AMNH). PERU Ayacucho: Monterico (PAN). Cajamarca: Nancho, Quebrada (MCZ); Fundo Sinchono, Cordillera Azul (AMNH); Monsón Valley, Tingo María (CAS). Junín: Amable María (PAN). Maraynioc (PAN); Loreto: Yarina Cocha (IRSNB). Madre de Dios: Rio Estari, ca. $13^{\circ} \mathrm{S}, 71^{\circ} \mathrm{W}$, o (MUSM). PANAMA Chiriquí: Volcán (MCZ). Panamá: Very common. Colón: Very common. TRINIDAD Simla Biological Station, Arima Valley (AMNH). VENEZUELA Aragua: Maracay (AMNH).

\section{Acacesia yacuiensis NEW SPECIES}

Figures 17-22; Map 1

Female holotype from Arroyo Yacui, Parque Nacional Iguazu, Misiones Province, Argentina, Jan. 1966 (M. E. Galiano) in MACN. Male paratype from Baixo Jordão, Siderópolis, Santa Catarina State, Brazil, Dec. 6, 1977 (F. R. Meyer), in MCN 7746. 
Note. The species is named after the collecting locality of the type specimen. Since males and females were never collected together, separate descriptions of coloration have been maintained in case the two are members of different species.

Description. Female holotype. Carapace. Clypeus tan. Chelicerae light tan. Labium, endites, sternum tan. Legs tan. Shape of abdomen quite similar to that of $A$. cornigera with narrow "waist" opening into protruding "shoulders." Brown shoulder patches, folium with orange spots on either side of the dagger shape. Dorsal branching pattern accentuated by two additional parallel lines on either side of main center line. Folium and outside of two of the three specimens handsome oxidized-copper color but third specimen dull tan. (It is impossible to determine which of these color schemes is most close to live coloration). Spotted appearance with no solid patches of color (Fig. 19). Sides gray-brown, venter graybrown, with no distinctive spots, unlike A. villalobosi. PME 0.7 diameters of AME, posterior LE 0.5 diameters, anterior LE 0.4 diameters. AME 1.3 diameters apart, 3.0 from LE. PME 1.4 diameters apart, 5.0 from LE. LE less than 1 diameter apart. Total length $7.9 \mathrm{~mm}$. Carapace length $2.5 \mathrm{~mm}$, width 2.2 , height 1.4. Carapace width behind posterior lateral eyes $1.1 \mathrm{~mm}$. First femur $3.8 \mathrm{~mm}$, patella-tibia 4.4, metatarsus 3.5, tarsus 1.4 . First leg length overall 13.1. Second patella-tibia $5.1 \mathrm{~mm}$, third 2.5 , fourth 3.8 .

Male paratype. Carapace yellow-brown. Clypeus light brown. Chelicerae light yellow-white. Labium light yellow-white, endites light yellow-white. Sternum light yellow-white. Coxae light yellow-white; legs light yellow-brown. Dorsum with dark gray-brown shoulder patches, off-white outside folium, light gray within, with parallel orange-brown dots surrounding the dagger which shades from gray to off-white. Branching pattern absent in the type, although the other specimens had visible branching. Sides light gray-brown. Venter light gray with two white ovoid patches onethird of the way up the venter on either side of spinnerets, as in $A$. villalobosi. PME 0.6 diameters of AME, anterior LE 0.4 diameters, posterior LE 0.3 diameters. AME 1.4 diameters apart, 1.3. from LE. PME 2.0 diameters apart, 2.2 from LE. LE less than 1 diameter apart. Total length $4.7 \mathrm{~mm}$. Carapace length $2.3 \mathrm{~mm}$, width 1.8 , height 1.1. Carapace width behind posterior lateral eyes $0.83 \mathrm{~mm}$. First femur $3.6 \mathrm{~mm}$, patella-tibia 3.9, metatarsus 2.5, tarsus 0.76 . 
First leg length overall 10.76. Both second legs were broken off below the patella; third patella-tibia $1.3 \mathrm{~mm}$, fourth $2.5 \mathrm{~mm}$.

Variation. Total length of females 5.3 to $8.6 \mathrm{~mm}$, males 4.3 to $4.7 \mathrm{~mm}$.

Diagnosis. The distinct possibilty remains that the types belong to two different species, with mismatched male and female. The male closely resembles $A$. villalobosi, but its paracymbium helps differentiate it. The proximal lobe of the paracymbium (Fig. 21) is a protruding thorn-shape while that of A. villalobosi (Fig. 33) is continuous with, and an integral part of, the paracymbium. Furthermore, while both A. yacuiensis and A. villalobosi have one fork of the median apophysis of the palpus markedly longer than the other, A. yacuiensis notably possesses a third lobe (Fig. 20). If the highly ornate dorsal pattern of the female (Fig. 19) is constant (there are very few specimens from which to make generalizations), then it should readily serve to distinguish A. yacuiensis from all the other species. It may be distinguished from A. cornigera by the additional parallel lines surrounding the branching pattern in the folium. The scape of the epigynum (Fig. 17), which is shorter than the base, sets A. yacuiensis apart from the other species where the scape is at least as long as the base. Although the sclerotized edges of the lateral plates (Fig. 18) are similar to those of A. cornigera (Fig. 10), this species lacks the keel on the median plate which appears in A. cornigera.

Specimens examined. ARGENTINA Misiones: Parque Nacional Iguazu, Arroyo Yacui, Jan. 1966, 2o (M. E. Galiano, MEG). BRAZIL Minas Gerais: Lavras. 18 Oct. 1978, ơ (W. D. Frank, MCZ). São Paulo: Municipio de Itu, Fazenda Pau d'Alho, 2 Feb. 1959, @ (F. Lane, AMNH); São Francisco Xavier, Serra Mantiqueira, Dec. 1944, ơ (E. Dente, MZSP 11059). Santa Catarina: Siderópolis, Baixo Jordão, 6 Dec. 1977, ơ (F. R. Meyer, MCN 7746).

\section{Acacesia benigna NEW SPECIES}

Figures 23-27; Map 1

Female holotype from Puesto de Vigilânte Pakitza, Zona Reservada de Manu, Depto. Madre de Dios, Peru, (D. Silva D. and J. Coddington), MUSM. Male paratype from Est. Biol. Beni, 
Depto. Beni, Bolivia, (J. Coddington et al.), USNM. The species name is the Latin adjective "friendly" the first four letters of which are a pun on the collecting site of the male paratype.

Description. Female holotype. Carapace light tan. Clypeus tan. Chelicerae light tan. Labium light yellow, endites tan. Sternum light yellow. Coxae light yellow; legs tan. Abdomen a pointed oval. Anterior portion of abdomen terminating in a hump which is apex of the dagger. Dorsum with light gray shoulder patches, offwhite outside slightly sinuous folium, slightly darker within, and a parallel row of brown dots on either side of the dagger, which is straight-sided and terminates in a long point. Branching pattern as in the other species. Line extends from root to base of abdomen. Sides light gray. Venter with light gray stripe extending from book lungs to spinnerets, flanked by off-white patches. PME 1.0 diameters of AME, LE 0.6 diameters. AME 2.0 diameters apart, 1.8 from LE. PME 1.5 diameters apart, 2.8 from LE. LE less than 1 diameter apart. Total length $6.6 \mathrm{~mm}$. Carapace length $2.5 \mathrm{~mm}$, width 1.8 , height 1.4. Carapace width behind posterior lateral eyes $0.9 \mathrm{~mm}$. First femur $4.4 \mathrm{~mm}$, patella-tibia 5.3, metatarsus 3.7, tarsus 1.2. First leg length overall 14.6. Second patella-tibia $4.3 \mathrm{~mm}$, third 2.0, fourth 3.2.

Male paratype. Color as in female. PME 0.6 diameters of AME, posterior LE 0.5 diameters, anterior LE 0.4 diameters. AME 1.0 diameters apart, 0.8 from LE. PME 2.0 diameters apart, 4.0 from LE. LE less than 1 diameter apart. Total length $6.5 \mathrm{~mm}$. Carapace length $2.5 \mathrm{~mm}$, width 2.7. Both first legs were broken off and missing in this specimen. Second patella-tibia $3.2 \mathrm{~mm}$, third 1.7, fourth 2.3.

Variation. Total length of females 5.9 to $8.8 \mathrm{~mm}$, of males, 5.0 to 6.5 .

Diagnosis. Dorsal pattern (Fig. 25) similar to A. yacuiensis or A. villalobosi. The epigynum seen from below (Fig. 23) is reminiscent of A. cornigera with elongate scape with slight lip, and underlying structures visible on either side of the scape. From beneath, the lateral plates of the epigynum are quite distinctive. The sclerites point outwards like reversed parentheses )( and resemble a Mickey Mouse hat which has big ears at the top (Fig. 24). As in A. cornigera, the paracymbium is higher than wide. In this species, 
though, the proximal lobe of the paracymbium is much closer in height to the distal lobe, with the two usually almost of equal length (Fig. 27). The tines of the median apophysis (Fig. 26) are equal in length.

Distribution. The limited number of specimens are from the border of eastern Peru and western Bolivia, with a single specimen from Brazil.

Specimens examined. BOLIVIA Beni: Est. Biol. Beni, Nov. 1989, o (J. Coddington et al., USNM); $16.8 \mathrm{mi} \mathrm{SW}$ Yucumo, 15-19 Nov. 1989, ơ, o (J. Coddington et al., USNM). La Paz: Coroico, 4 Nov. 1989, ơ (J. Coddington et al., USNM). BRAZIL Pará: Aldeia Aracu, Gurupi-Uma, 50 km E. Canindé, Rio Gurupi. 2-30 May 1963, ơ (B. Malkin AMNH). PERU Amazonas: alto Rio Comaina, Puesto de Vigilancia 22 "Falso Paquisha," 850-1,150 m, 21 Oct.-3 Nov. 1987, 2@ (D. Silva D., MUSM). Loreto: Iquitos, May 1920, o (H. S. Parrish, MCZ). Madre de Dios: Zona Reservada de Manu, Puesto de Vigil, Pakitza, 30 Sept. 1987, ९, 24 Sept.-10 Oct. 1987, ơ, 6 Oct. 1987, ९, 26 Sept. 1987, ơ (J. Coddington, D. Silva D., MUSM); Albergue "Cuzco Amazonico," 22 Feb.-8 Mar. 1990, ९९, Ơ ${ }^{\circ}$, 24 June 1989, ơ (D. Silva D., MUSM); Zona Reservada Tambopata, Trocha de los Terrazos, 20 Aug. 1987, ơ, 22 May 1988, ơ (D. Silva D., MUSM); Zona Reservada da Tambopata, Trocha Puesto del Sol, 9 June 1988, ơ (D. Silva D., MUSM). Pasco: Huancabamba, Quebrada Chispa, NW de Iscozacin, 345m, 1 Nov. 1986, ơ (D. Silva D., MUSM). Ucayali: Pucallpa, 7 Nov. 1946, ९ (J. C. Pallister, AMNH).

\section{Acacesia villalobosi NEW SPECIES}

Figures 28-33; Map 1

Female holotype from Serra dos Orgãos, Rio de Janeiro State, Brazil, collected in forest, 1,000-1,800m, 19 April 1965 (H. W. Levi), in MZSP ex MCZ. Male paratype from Guarulhos, São Paulo State, Brazil, 9 Sept. 1962 (R. France), in MZSP. The species is named for the Brazilian composer Heitor Villa-Lobos.

Description. Female holotype. Carapace yellow-brown. Clypeus yellow-brown. Chelicerae yellow-brown. Labium yellow-white, endites yellow-white. Sternum yellow-brown. Coxae yellowbrown; legs yellow-brown. Dorsum of abdomen light gray inside 
folium, with several parallel orange spots, off-white outside wiggly-lined folium, black shoulder patches; sides dark gray, venter dark gray, with two oblong white patches on the venter three-quarters of the distance between epigynum and spinnerets, spaced on either side of spinnerets. PME 1.1 diameters of AME, LE 0.5 diameters. AME 1.4 diameters apart, 1.8 from LE. PME 2.1 diameters apart, 5.0 diameters from LE. LE less than 1 diameter apart. Total length $6.1 \mathrm{~mm}$. Carapace length $2.2 \mathrm{~mm}$, width 1.7 , height 1.3. First femur $3.6 \mathrm{~mm}$, patella-tibia 4.3, metatarsus 2.9 , tarsus 1.2 . First leg length overall 12.0. Second patella-tibia $3.7 \mathrm{~mm}$, third patella-tibia 1.8, fourth 2.8. Note: first right leg was regenerating.

Male paratype from Brazil, Campo do Jordão, São Paulo State, MZSP. Color as in female. Two orange-brown faint parallel lines lead from tip of dagger down to bottom of the folium. PME 0.8 diameters of AME, LE 0.4 diameters. AME 1.3 diameters apart, 1.1 from LE. PME 1.9 diameters apart, 3.0 diameters from LE. LE less than 1 diameter apart. Total length $4.9 \mathrm{~mm}$. Carapace length $2.2 \mathrm{~mm}$, width 1.9 , height 0.65 . Carapace width behind posterior lateral eyes $0.8 \mathrm{~mm}$. First femur $3.5 \mathrm{~mm}$, patella-tibia 4.0, metatarsus 2.9, tarsus 0.94. First leg length overall 11.34. Second patellatibia 2.9, third $1.4 \mathrm{~mm}$, fourth 2.3 .

Variation. There are only six male specimens of this species known. Total length of ten females 4.3 to $6.1 \mathrm{~mm}$, of the six males, 3.7 to 5.0 .

Diagnosis. Acacesia villalobosi is most recognizable by the distinct oval patches on its venter (Fig. 31), which occur in both male and female. While the dorsal pattern and shape of the abdomen are almost identical to that of $A$. cornigera, many specimens could be distinguished by the additional parallel lines within the folium (Fig. 30). The female's distinctively wide, trapezoidal-shaped epigynum (Fig. 28) is an almost fail-proof character and its sclerites, shaped like commas on their sides (Fig. 29), also differ sufficiently from A. hamata and A. cornigera for easy diagnosis. The males' palpi are notable both for the extreme length of the prongs of the median apophysis and for the shape of the paracymbium (Fig. 33). The claw of the median apophysis is long such that a cursory glance gives the impression that there is only one projection, instead of two. Also notable is the line just beyond the tip of the embolus (Fig. 32). 
Distribution. The species has been collected along the southeastern coast and inland of Brazil.

Specimens examined. BRAZIL Espírito Santo: Lacerda [= Loanda?], ९ (MNRJ). Minas Gerais: Serra do Caraça, o; 24 Feb.-3 Mar. 1972, o (Martins, Demet, Vanin, MZSP 10.809 ). Rio de Janeiro: Serra dos Orgãos, 19 Apr. 1965, ○ (H. W. Levi, MCZ); Teresópolis, o (AMNH). Rio Grande do Sul: Fazenda Aver, Bom Jesus, 24 Mar. 1989, ९ (A. B. Bonaldo, MCN 18422); Cambara do Sul, Fortaleza dos Aparados, 5 Jan. 1978, ९ (P. C. Braun, MCN); Vila Oliva, 10 Jan. 1986, ९९ (P. A. Buckup, MCN 3737); Canela, 26 Dec. 1975, ९ (A. Lise, MCN 3693); Sobradinho, 10 Feb. 1985, ९ (A. Lise, MCN 12885); Novo Hamburgo, 4 Nov. 1985, ९ (C. J. Becker, MCN 14354); Morro Santana, Porto Alegre, 28 Oct. 1985, @ (R. A. Ramos, MCN 18976). Santa Catarina: Pinhal, May 1948, ९; Dec. 1948, @ (A. Maller, AMNH). São Paulo: São Paulo, @ (AMNH); Boraceia, ९ (MZSP); Faz. Sta. Maria, Amparo, 28 Nov. 1942, ९९, ơ, imm. (F. Lane, MZSP 10.808); S. Jose Barreiro, S. Bocaina, Nov. 1968, ơ (̛ (M. Alvarenga, AMNH); Mata do Governo, Inst. Botanica. 4 Mar. 1959, \& (L. Lane, AMNH); Parque Municipal de Botucatu, 16 Dec. 1986, ९ (I. M. P. Rinaldi, L. C. Forti, IMPR); Municipio de Itu, Fazenda Pau d'Alho, 2 Feb. 1959 ९९ (F. Lane, AMNH); Camino de Mar, $33 \mathrm{~km} \mathrm{~S}$ of São Paulo, 11 Apr. 1965, ९ (H. Levi, P. de Biasi, MCZ); Campo do Jordão, 3 Jan. 1948, ९, ర̛ (̛ (F. Lane, MZSP 1102); Campo do Jordão, Pico de Itapeva, ९ (MZSP); Alto da Serra, 15 Dec. 1951, ९९, ơ (W. Bakermann, MZSP 7517); Fazenda Intervales, 15 km E Guapiara, Feb. 1990, o (W. Eberhard, MCZ). ?Mato Grosso: Sta. Rita Caldas Dec. 1953, ९ (P. Pereira, MZSP).

\section{ACKNOWLEDGEMENTS}

I wish to thank Herbert W. Levi for making my project possible and for his invaluable instruction in every aspect of taxonomy from biological illustration to literature. Without his guidance and examples this revision would have been unfeasible. He also provided the illustrations for Figures 1-3 of an expanded palpus, macroseta, and paracymbium. Laura Leibensperger helped with the minutiae of computing, labeling, and using the microscope, and contributed further species identifications to the list I had compiled. William Piel and Edward Cutler offered philosophical and 
intellectual insights that helped me clarify my position. David Furth made suggestions on biogeography; both he and Kenneth Boss made recommendations. Ardis Johnston's much-needed advice greatly improved the appearance of this paper in the finishing flurry of activity.

Specimens were made available by the following institutions and individuals:

AMNH American Museum of Natural History, New York; N. Platnick, L. Sorkin

BMNH The Natural History Museum, London, United Kingdom; P. Hillyard

CAS California Academy of Sciences, San Francisco; W. J. Pulawski, D. Ubick; includes Exline-Peck collection

IRSNB Institut Royal des Sciences Naturelles de Belgique, Brussels, Belgium; L. Baert

MACN Museo Argentino de Ciencias Naturales, Buenos Aires, Argentina; E. A. Maury

MCN Museu de Ciências Naturais, Porto Alegre, Rio Grande do Sul, Brazil; E. H. Buckup

MCZ Museum of Comparative Zoology, Cambridge, Massachusetts

MEG M.E. Galiano, Buenos Aires, Argentina

MUSM Museo de Historia Natural, Universidad Nacional Mayor de San Marcos, Lima, Peru; D. Silva D.

MNHN Museum National d'Histoire Naturelle, Paris; J. Heurtault, C. Rollard

MNRJ Museu Nacional, Rio de Janeiro, Brazil; A. Timotheo da Costa

MZSP Museu de Zoologia da Universidade de São Paulo, São Paulo, Brazil; P. Vanzolini, L. Neme, J. L. M. Leme

PMY Peabody Museum, Yale University, New Haven; D. Furth

IMPR I. M. P. Rinaldi, Botucatu, São Paulo, Brazil

USNM National Museum of Natural History, Smithsonian Institution, Washington, D.C.; J. Coddington

UCV Universidad Central de Venezuela, Caracas, Venezuela; J. Racenis

ZMK Zoologisk Museum, København, Denmark; H. Enghoff 


\section{REFERENCES CITED}

Badcock, H. D. 1932. Arachnida from the Paraguayan Chaco. Journal of the Linnean Soc. London (Zoology) 38: 1-48.

Bonnet, P. 1955. Bibliographia Araneorum. Vol. 2. Toulouse: Douladoure.

Brignoli, P. M. 1983. A catalogue of the Araneae described between 1940 and 1981. Manchester University Press.

Bryant, E. 1945. The Argiopidae of Hispaniola. Bull. Mus. Comp. Zoology 95(4): 359-418.

Cambridge, F. O. P.- 1897-1905. Biologia Centrali-Americana. vol. 2: Arachnida, Araneidea and Opiliones. London.

Caporiacco, L. di 1954. Araignées de la Guyane Française du Museum d'Histoire Naturelle de Paris. Comment. Pontificia Acad. Scient. 16(3): 45-193.

- 1955. Estudios sobre los Aracnidos de Venezuela. Acta Biol. Venezuelica, 1: 251-265.

Chamberlin, R. V. 1916. Results of the Yale Peruvian expedition of 1911. Bull. Mus. Comp. Zool., 60: 177-299.

Chamberlin, R. V., and Ivie, W. 1936. New spiders from Mexico and Panama. Bull. Univ. Utah (Biol. Ser.) 3(5): 1-104.

Chickering, A. M. 1955. The genus Eustala (Araneae, Argiopidae) in Central America. Bull. Mus. Comp. Zool. 112: 391-518.

Comstock, J. H. 1912. The spider book. Garden City, New York: Doubleday, Page and Company.

- 1940. The spider book. Rev. ed. New York: Doubleday, Doran and Company.

Eberhard, W. 1985. Sexual selection and animal genitalia. Cambridge: Harvard University Press.

Emerton, J. H. 1884. New England spiders of the family Epeiridae. Trans. Connecticut Acad. Scienc. 6: 295-342.

Foelix, R. F. 1982. Biology of spiders. Cambridge: Harvard University Press.

Hentz, N. 1847. Descriptions and figures of the Araneides of the United States. Boston J. Natur. Hist. 5: 443-479.

- 1875. The spiders of the United States: A collection of the arachnological writings of Nicholas Marcellus Hentz, M.D. Ed. Edward Burgess. Boston: Boston Society of Natural History.

Kaston, B. J. 1948. Spiders of Connecticut. Bull. Connecticut Geol. Natur. Hist. Surv. 70. Hartford.

- 1978. How to know the spiders. 3d edition. Dubuque: Wm. C. Brown Co. Publishers.

Keyserling, E. 1865. Beiträge zur Kenntnis der Orbitelae Latr. Abhand. zoolog.-bot. Gesell. Wien 14: 799-856.

Levi, H. W. 1976. The orb-weaver genera Verrucosa, Acanthepeira, Wagneriana, Acacesia, Wixia, Scoloderus and Alpaida north of Mexico (Araneae: Araenidae). Bull. Mus. Comp. Zool. 147(8): 351-391.

Marx, G. 1890. Catalogue of the described Araneae of temperate North America. Proc. U.S. Nation. Mus. 12(782): p.497-594.

Mayr, E. 1969. Principles of systematic zoology. New York: McGraw Hill.

Mayr, E., and Ashlock, P. 1991. Principles of systematic zoology. 2d ed. New York: McGraw Hill. 
McCook, H. C. 1894. American spiders and their spinningwork. vol. 3. Philadelphia: Academy of Natural Sciences of Philadelphia.

Mello-Leitão, C. F. 1948. Contribuição ao conhecimiento da fauna araneologica da Guianas. An. Acad. Brasileira Cienc. 20(2): 151-196.

Petrunkevitch, A. 1911. A synonymic index catalogue of spiders of North, Central and South America. Bull. Amer. Mus. natur. Hist. 29: 1-809.

1925. Arachnida from Panama. Trans. Connecticut Acad. Arts Scienc. 27: $51-248$.

Roewer, C. Fr. 1942. Katalog der Araneae von 1758 bis 1940. Vol.1. Bremen.

Simon, E. 1892-1895. Histoire naturelle des Araigneés. vol. 1. 2d ed. Paris: Librairie Encyclopédique de Roret. 

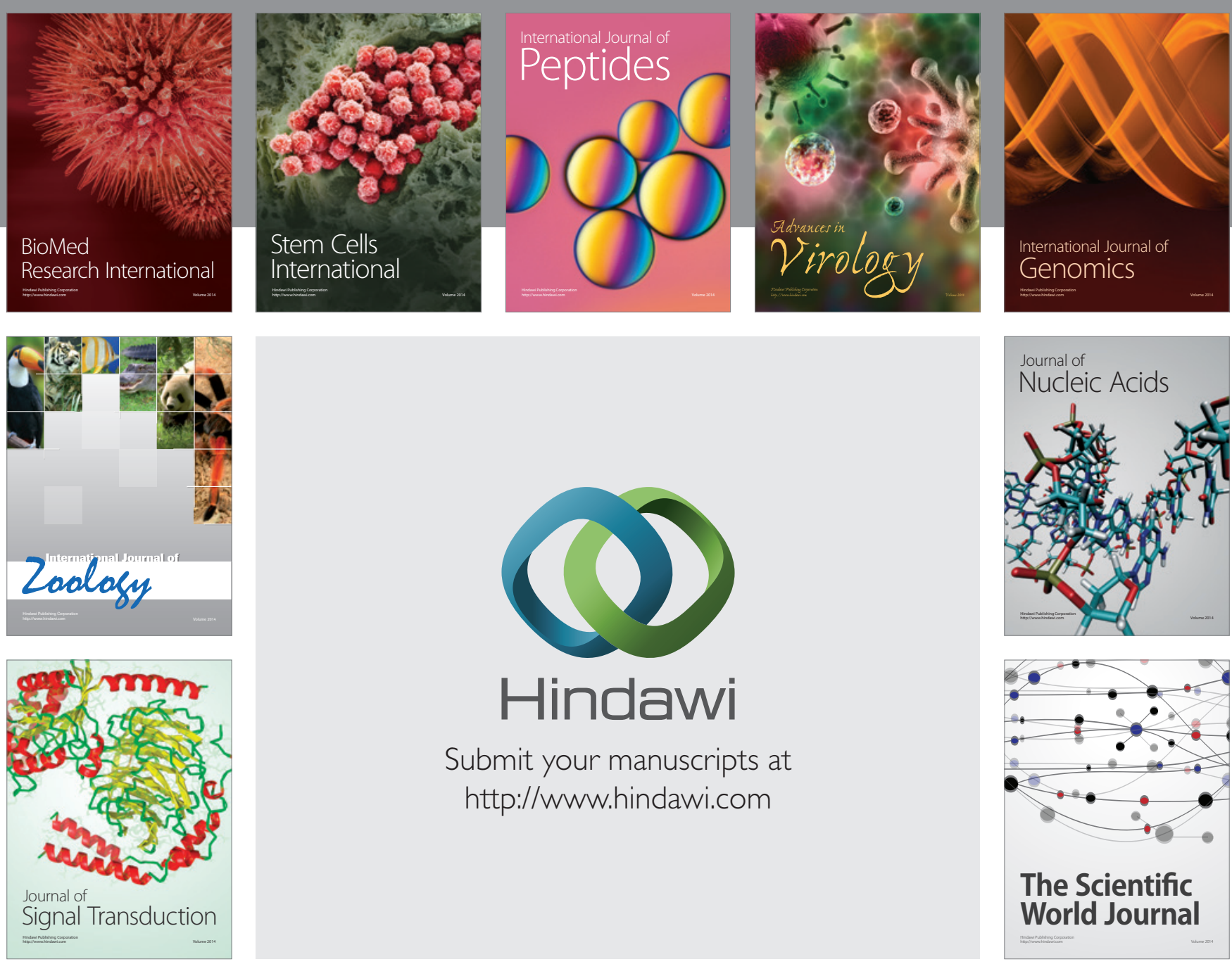

Submit your manuscripts at

http://www.hindawi.com
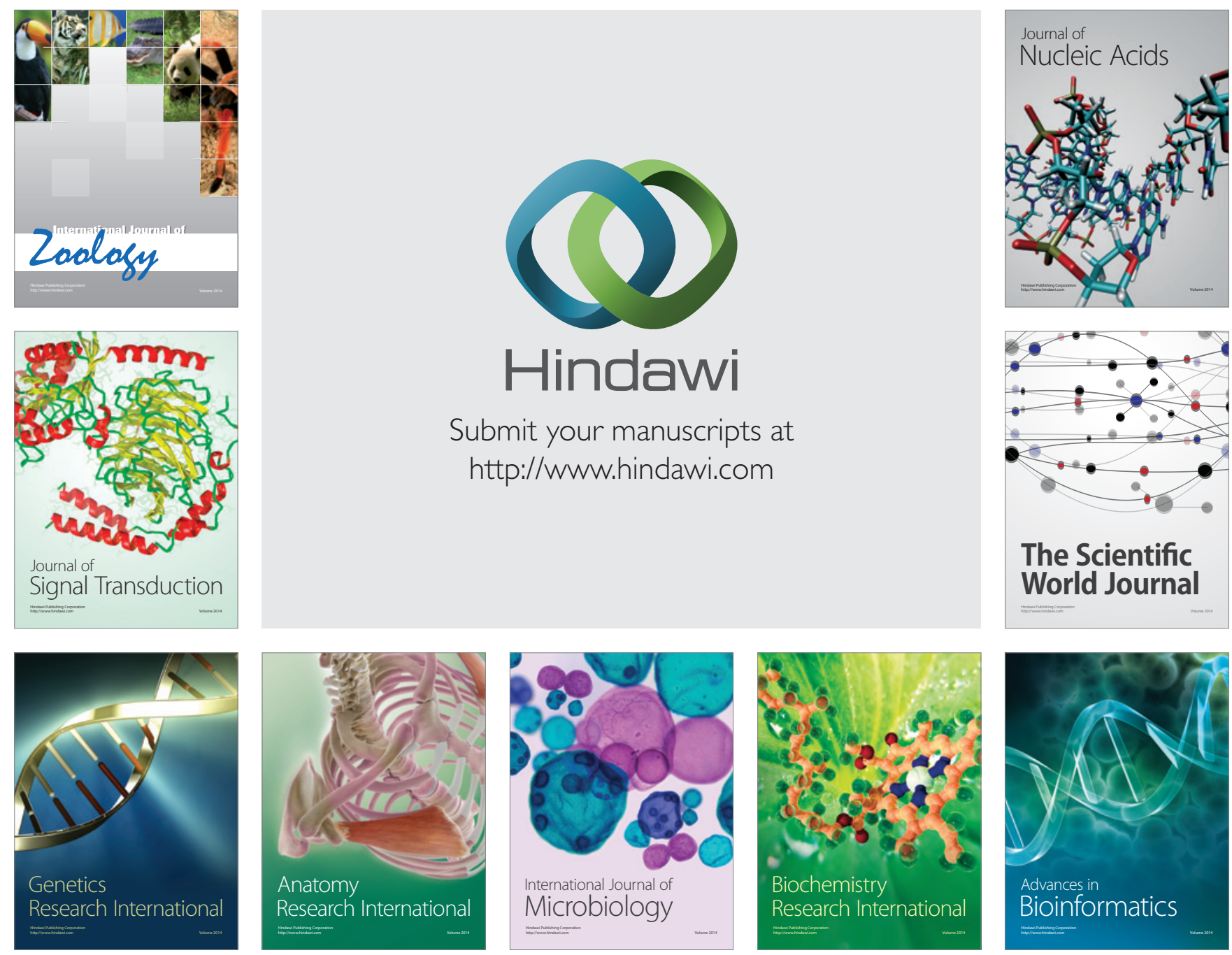

The Scientific World Journal
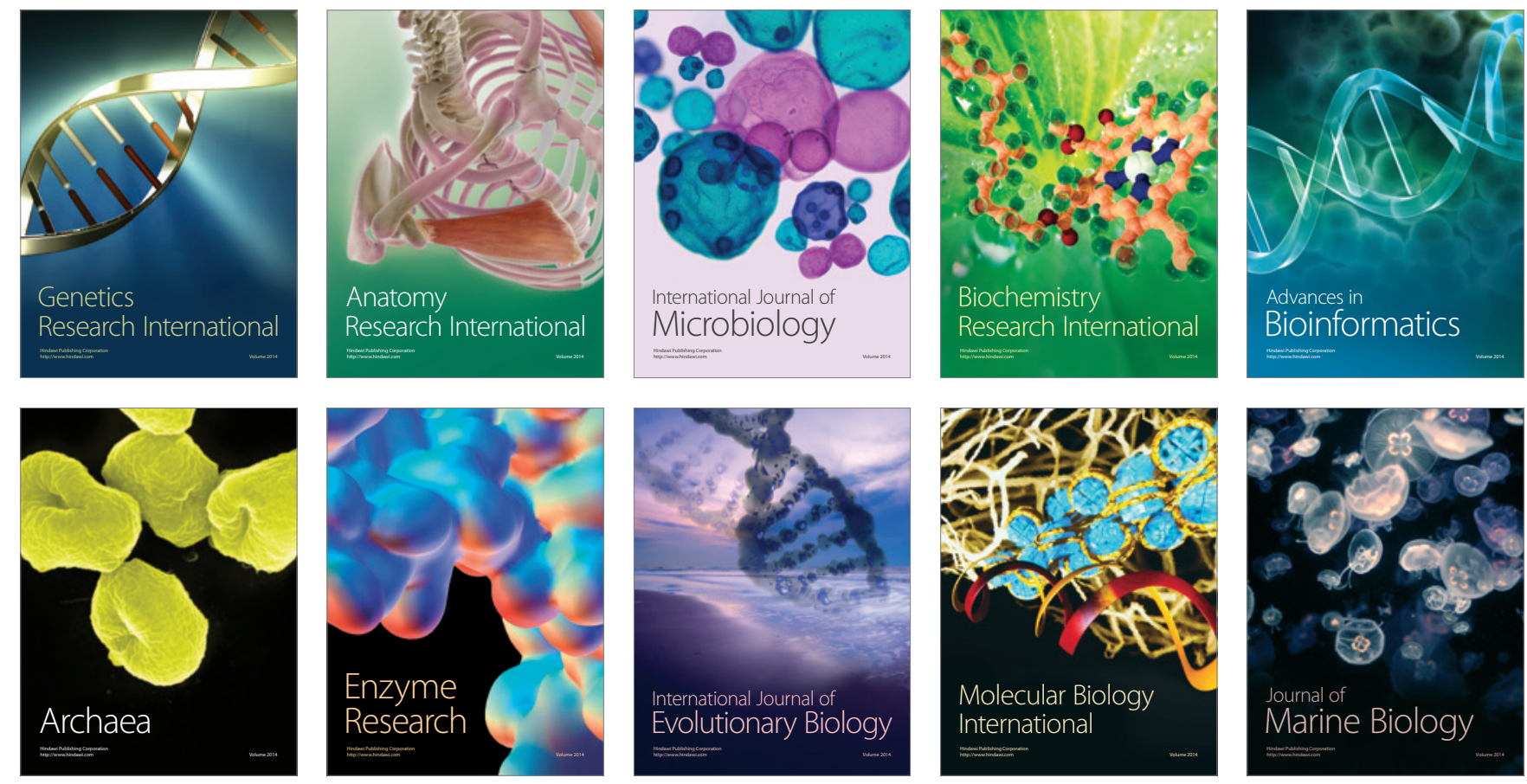